PHYSICAL REVIEW E 83, 041503 (2011)

\title{
Tests of mode-coupling theory in two dimensions
}

\author{
Fabian Weysser $^{1, *}$ and David Hajnal ${ }^{2}$ \\ ${ }^{1}$ Fachbereich Physik, Universität Konstanz, D-78457 Konstanz, Germany \\ ${ }^{2}$ Institut für Physik, Johannes Gutenberg-Universität Mainz, Staudinger Weg 7, D-55099 Mainz, Germany
}

(Received 13 January 2011; published 25 April 2011)

\begin{abstract}
We analyze the glassy dynamics of binary mixtures of hard disks in two dimensions. Predictions of the mode-coupling theory (MCT) are tested with extensive Brownian dynamics simulations. Measuring the collective particle density correlation functions in the vicinity of the glass transition, we verify four predicted mixing effects. For instance, for large size disparities, adding a small amount of small particles at a fixed packing fraction leads to a speedup in the long-time dynamics, while for small size disparities it leads to a slowing-down. Qualitative features of the nonergodicity parameters and the $\beta$ relaxation, which both depend in a nontrivial way on the mixing ratio, are found in the simulated correlators. Studying one system in detail, we are able to determine its ideal MCT glass transition point as $\varphi^{c}=0.7948$ and test MCT predictions quantitatively.
\end{abstract}

DOI: 10.1103/PhysRevE.83.041503

PACS number(s): 64.70.P-, 64.70.Q-, 82.70.Dd

\section{INTRODUCTION}

Simulations of simple model systems provide a powerful and important means for studying the interesting phenomenon known as glass transition [1]. Considering a binary mixture of hard disks close to vitrification in a computer simulation unveils the opportunity to investigate a system that is simple enough for the particle interactions to be described theoretically but exhibits the full range of glassy behaviors as autocorrelation functions with two-step relaxation processes showing divergent relaxation time scales upon approaching the glass transition.

In 1984, the mode couping theory (MCT), which is a microscopic theory for glassy dynamics, was introduced by Bengtzelius, Götze, and Sjölander [2] and Leutheusser [3]. It was able to correctly predict many features of the complex dynamics of glass-forming liquids and was studied in great detail by Götze and coworkers (see Refs. [4,5] for detailed reviews). The basic version of MCT considers isotropic and homogeneous one-component liquids in three spatial dimensions (3D). The only model-dependent input is given by the static structure factors of the considered liquid. The most prominent prediction of MCT is a dynamic transition from a liquid into an ideal nonergodic glassy state at some critical temperature or particle density.

In reality, one-component (monodisperse) systems do not serve as good glass formers, since they tend to form crystals rather than amorphous solids. Crystallization can be suppressed by using polydisperse systems. The simplest polydisperse system is a binary mixture. Since it is well known that adding a second component to a one-component liquid may strongly influence both its static and its dynamic properties, Götze and Voigtmann [6] have systematically investigated the glass transition behavior of binary hard spheres in 3D. They have found four mixing effects: (i) small size disparities stabilize the glass, (ii) large size disparities stabilize the liquid, increasing the concentration of the smaller particles leads to both (iii) an increase in the plateau values

*Corresponding author: fabian.weysser@uni-konstanz.de of the normalized correlation functions for intermediate times for wave numbers that are not too small and (iv) a slowing down of the relaxation of the correlators of the bigger particles towards their plateaus. These results qualitatively agree with those from dynamic light-scattering experiments [7-9] and molecular dynamics simulations $[10,11]$.

Several physical phenomena such as equilibrium phase transitions strongly depend on the spatial dimensionality $\mathcal{D}$. Thus, the question of the $\mathcal{D}$ dependence of the glass transition naturally arises. From a fundamental point of view, there are interesting studies concerning glass transitions in high dimensions; see, for instance, the recent publications of Schmid and Schilling [12] or Ikeda and Miyazaki [13], and references therein. In the present paper, we restrict ourselves to the case $\mathcal{D}=2$. An experimental realization of a model glass former in two dimensions (2D) was presented by Ebert et al. [14]. They considered binary mixtures of superparamagnetic colloidal particles confined at a water-air interface that interact via repulsive dipole potentials. The magnetic moments are induced by an external magnetic field perpendicular to the water interface. The results for time-dependent correlation functions measured by video microscopy clearly exhibit slow glassy dynamics as found by König et al. [15] and Mazoyer et al. [16]. Computer simulation results of Santen and Krauth [17] for polydisperse hard disks in 2D also give evidence of the existence of a dynamic glass transition in 2D. Bayer et al. [18] explored the question of the $\mathcal{D}$ dependence of the glass transition by solving the mode-coupling equations for a one-component system of hard disks in 2D. They found an ideal glass transition. On a qualitative level, the results of Bayer $e t$ al. for the glass transition scenario for monodisperse hard disks in 2D are very similar to the corresponding MCT results of Franosch et al. [19] for one-component systems of hard spheres in 3D.

The MCT study by Bayer et al. [18] was extended to binary mixtures of hard disks by Hajnal et al. [20] and also to binary mixtures of dipolar particles in 2D [21]. For binary hard disks in 2D the same four mixing effects occur that have been reported before by Götze and Voigtmann [6] for binary mixtures of hard spheres in 3D. Furthermore, it was shown that the glass transition diagram for binary hard disks in $2 \mathrm{D}$ 
strongly resembles the corresponding random close packing diagram. This fact is a hint for the applicability of the MCT approximations in $2 \mathrm{D}$.

So far, the MCT results of Hajnal et al. [20] for the dynamics of binary hard disks in 2D have not yet been tested systematically within the framework of atomistic computer simulations. To fill in this gap is the main motivation of our contribution. For this purpose we perform Brownian dynamics (BD) simulations for binary mixtures of hard disks in 2D. First, we verify the existence of the four mixing effects predicted by MCT that are briefly described above. Second, we present a quantitative comparison of time-dependent collective density correlators from MCT and our BD simulations.

To achieve a self-contained presentation, we have organized the paper as follows: in Secs. II and III we introduce the correlation functions of central interest and we define our model system. In Sec. IV we describe our BD simulation techniques. Section V contains a brief review of the equations and central predictions of MCT. We present our results in Sec. VI. We summarize and conclude in Sec. VII.

\section{PRELIMINARIES}

\section{A. Matrix algebra}

In the following, we make use of the compact mathematical notation introduced in Ref. [20]. Bold symbols $\boldsymbol{A}, \boldsymbol{B}$, etc., denote arrays of $M \times M$ matrices whose components $\boldsymbol{A}_{k}, \boldsymbol{B}_{k}$ are labeled with subscript Latin indices. Their elements $A_{k}^{\alpha \beta}$, $B_{k}^{\alpha \beta}$, also denoted $(\boldsymbol{A})_{k}^{\alpha \beta},(\boldsymbol{B})_{k}^{\alpha \beta}$, are indicated by superscript Greek indices. Matrix products are defined componentwise, for example, $\boldsymbol{C}=\boldsymbol{A} \boldsymbol{B}$ means $\boldsymbol{C}_{k}=\boldsymbol{A}_{k} \boldsymbol{B}_{k}$ for all $k . \boldsymbol{A}$ is called positive (semi-)definite $(\boldsymbol{A} \succeq \mathbf{0}) \boldsymbol{A} \succ \mathbf{0}$ if this is true for all $\boldsymbol{A}_{k}$. For discretized model systems where $k$ is restricted to a finite number of values, we define the standard scalar product $(\boldsymbol{A} \mid \boldsymbol{B})=\sum_{k} \sum_{\alpha, \beta}\left(A_{k}^{\alpha \beta}\right)^{*} B_{k}^{\alpha \beta}$, where the superscript asterisk stands for complex conjugation. The standard norm of $\boldsymbol{A}$ is then given by $|\boldsymbol{A}|=\sqrt{(\boldsymbol{A} \mid \boldsymbol{A})}$.

\section{B. Density correlators}

We consider an isotropic and homogeneous classical fluid consisting of $M$ macroscopic components, each containing $N_{\alpha}$ particles of a species $\alpha$ in $\mathcal{D}$ spatial dimensions. The total number of particles in the system is then given by $N=\sum_{\alpha=1}^{M} N_{\alpha}$. Let $n^{\alpha}(\vec{r}, t)=\sum_{i=1}^{N_{\alpha}} \delta\left[\vec{r}-\vec{r}_{\alpha, i}(t)\right]$ denote the time-dependent microscopic particle density of the component $\alpha$ of the liquid, where $\vec{r}_{\alpha, i}(t)$ is the position of particle $i$ of component $\alpha$ at time $t$ and $\delta[\cdot]$ is the Dirac $\delta$ distribution. The time-dependent density fluctuation of the component $\alpha$ of the liquid at wave vector $\vec{k} \neq \overrightarrow{0}$ is given by the spatial Fourier transform,

$$
n_{\vec{k}}^{\alpha}(t)=\sum_{i=1}^{N_{\alpha}} \exp \left[i \vec{k} \cdot \vec{r}_{\alpha, i}(t)\right]
$$

of the particle density $n^{\alpha}(\vec{r}, t)$. We focus our discussion on the matrix $\boldsymbol{\Phi}(t)$ of time-dependent partial autocorrelation functions of density fluctuations, which provide a statistical description of a multicomponent liquid. For $t \geqslant 0$, its components at wave number $k$ are defined by the expressions

$$
\begin{aligned}
\Phi_{k}^{\alpha \beta}(t)= & \left\langle N^{-1}\left[n_{\vec{k}}^{\alpha}(t)\right]^{*} n_{\vec{k}}^{\beta}(0)\right\rangle_{\mathrm{TL}}, \quad k>0, \\
& \Phi_{0}^{\alpha \beta}(t)=\lim _{k \rightarrow 0^{+}} \Phi_{k}^{\alpha \beta}(t),
\end{aligned}
$$

where $\langle\ldots\rangle_{\mathrm{TL}}$ stands for canonical averaging followed by carrying out the thermodynamic limit. The zero-time value of the correlation matrix defines the normalization $\boldsymbol{\Phi}(0)=$ $\boldsymbol{S} \succ \mathbf{0}$, the positive-definite static structure factor matrix whose elements obey $\lim _{k \rightarrow \infty} S_{k}^{\alpha \beta}=x_{\alpha} \delta_{\alpha \beta}$. Here $\delta_{\alpha \beta}$ depicts the Kronecker delta, and $x_{\alpha}=N_{\alpha} / N$ the particle number concentration of the component $\alpha$.

\section{MODEL SYSTEM}

In this work we investigate binary mixtures of hard disks in 2D with diameters $d_{\alpha}$ that are distributed isotropically and homogeneously with total particle number density $n$. Consisting of "big" $(\alpha=b)$ and "small" $(\alpha=s)$ particles with diameters $d_{s} \leqslant d_{b}$, the system is coupled to a heat bath with thermal energy $k_{B} T$ and its dynamics is governed by Brownian motion. The masses $m_{\alpha}$ and the single-particle short-time diffusion coefficients $D_{\alpha}^{0}$ are set to $m_{s}=m_{b} \equiv m_{0}$ and $D_{s}^{0}=D_{b}^{0} \equiv D_{0}$, for simplicity.

It is well known that the thermodynamic equilibrium state of the considered model system depends on three independent control parameters. Making use of this implicitness, we choose them to be the total $2 \mathrm{D}$ packing fraction $\varphi=n(\pi / 4)\left(x_{b} d_{b}^{2}+\right.$ $\left.x_{s} d_{s}^{2}\right)$, the particle number concentration $x_{s}=N_{s} / N$ of the smaller disks, and the size ratio $\delta=d_{s} / d_{b}$.

\section{BROWNIAN DYNAMICS SIMULATION}

The basic concept of the algorithm has been described in detail in 3D in Ref. [22] and can easily be adapted to the 2D model specified above [23]. We consider binary mixtures of hard disks with size ratios of $\delta=d_{s} / d_{b} \in\{5 / 7,1 / 3\}$ with particle number concentrations $x_{s} \in\{0.4,0.5,0.6,0.7,0.8\}$. $N=1000$ hard disks move in a $2 \mathrm{D}$ simulation box of volume $V$ with periodic boundary conditions at packing fraction $\varphi$ as defined in Sec. III. After placing the particles in their initial positions we provide Gaussian-distributed velocities with variance $\left\langle\left|\vec{v}_{\alpha, i}\right|^{2}\right\rangle \equiv v_{0}^{2}$. To propagate the system at time $t$ forward in time, we employ a semi-event-driven algorithm. For every particle, for example, for particle $i$ of species $\alpha$ at time $t$, the algorithm determines the possible collision time $\Delta t_{i j}^{\alpha \beta}$ with any other particle. This is easily achieved by solving the equation

$$
\frac{d_{\alpha}+d_{\beta}}{2}=\left|\vec{r}_{i j}^{\alpha \beta}+\vec{v}_{i j}^{\alpha \beta} \Delta t_{i j}^{\alpha \beta}\right|,
$$

where $\vec{r}_{i j}^{\alpha \beta}=\vec{r}_{\beta, j}-\vec{r}_{\alpha, i}$ denotes the vector pointing from the center of particle $i$ of species $\alpha$ to the center of particle $j$ of species $\beta . \vec{v}_{i j}^{\alpha \beta}=\vec{v}_{\beta, j}-\vec{v}_{\alpha, i}$ denotes the corresponding relative velocity. The smallest solution $\Delta t=\min \left\{\Delta t_{i j}^{\alpha \beta}\right\}$ for all particle pairs determines the next event in the algorithm. All particles can then be propagated with constant velocity according to $\vec{r}_{\alpha, i}\left(t+t^{\prime}\right)=\vec{r}_{\alpha, i}(t)+\vec{v}_{\alpha, i} t^{\prime}$ for all $t^{\prime} \in[0, \Delta t]$. 
At time $t+\Delta t$, for two colliding particles the elastic binary collision laws impose new velocities:

$$
\begin{aligned}
\vec{u}_{\alpha, i} & =\vec{v}_{\alpha, i}+\left|\vec{r}_{i j}^{\alpha \beta}\right|^{-2}\left(\vec{r}_{i j}^{\alpha \beta} \cdot \vec{v}_{i j}^{\alpha \beta}\right) \vec{r}_{i j}^{\alpha \beta}, \\
\vec{u}_{\beta, j} & =\vec{v}_{\beta, j}-\left|\vec{r}_{i j}^{\alpha \beta}\right|^{-2}\left(\vec{r}_{i j}^{\alpha \beta} \cdot \vec{v}_{i j}^{\alpha \beta}\right) \vec{r}_{i j}^{\alpha \beta} .
\end{aligned}
$$

Due to the boundary conditions, any particle in the vicinity of the box boundary can collide with an image particle coming from the other end of the box with size $L=\sqrt{V}$.

So far, the algorithm described above yields ballistic motion. To mimic Brownian motion we modify it by introducing a thermostat that, at every integer-multiple of time $\tau_{B} v_{0} / d_{s}=0.01$, triggers a so-called Brownian step. In the Brownian step, all particle velocities are freshly drawn from a Gaussian distribution with variance $m_{0} v_{0}^{2} /\left(k_{B} T\right)=2$ for all particles. This assures that the particles move diffusively with a short-time diffusion coefficient $D_{0} /\left(v_{0} d_{s}\right)=0.005$ on time scales that are large compared to $\tau_{B}$.

As the system starts from a cubic lattice it is necessary to wait for the system to relax before meaningful stationary averages can be taken. Equilibration was performed with Newtonian dynamics (without imposing the Brownian step) for $10^{5}$ time steps in units of $d_{s} / v_{0}$. We assume that the system is equilibrated when the time-dependent correlation functions do not depend on the time origin. Correlation functions (with imposition of the Brownian time step) were measured in a time window of $10^{6}$ time steps in units of $d_{s} / v_{0}$, which is equivalent to 2551.02 time steps in units of $d_{b}^{2} / D_{0}$. The collective density correlation functions given by Eq. (2) can be calculated directly from the particle positions.

We selected systems with radius ratios $\delta=5 / 7$ and $\delta=$ $1 / 3$. Simulations were performed for $x_{s} \in\{0.1,0.2, \ldots, 0.9\}$, and the structure factors and radial distribution functions were compared with the Percus Yevick results, making it possible to exclude the ones with crystallization. For $\delta=$ $5 / 7$ and $x_{s} \in\{0.4,0.5,0.6,0.7\}$ and for $\delta=1 / 3$ and $x_{s} \in$ $\{0.5,0.6,0.7,0.8\}$, we found suitable candidates that are still amorphous at high packing fractions $\varphi$ and thus allow us to investigate the glassy behavior.

\section{MODE-COUPLING THEORY}

\section{A. Basic equations}

The MCT is based on the exact Zwanzig-Mori equation with subsequent application of the mode-coupling approximations [4]. For Brownian dynamics it reads

$$
\boldsymbol{\tau} \dot{\boldsymbol{\Phi}}(t)+\boldsymbol{S}^{-1} \boldsymbol{\Phi}(t)+\int_{0}^{t} \mathrm{~d} t^{\prime} \boldsymbol{m}\left(t-t^{\prime}\right) \dot{\boldsymbol{\Phi}}\left(t^{\prime}\right)=\mathbf{0} .
$$

The components of the matrix of microscopic relaxation times $\tau$ shall be approximated by $\tau_{k}^{\alpha \beta}=\delta_{\alpha \beta} /\left(k^{2} D_{\alpha}^{0} x_{\alpha}\right)$, where $D_{\alpha}^{0}$ is the single-particle short-time diffusion coefficient of a tagged particle of species $\alpha$ inside the fluid. MCT approximates the memory kernel $\boldsymbol{m}(t)$ by a symmetric bilinear functional,

$$
\boldsymbol{m}(t)=\mathcal{F}[\boldsymbol{\Phi}(t), \boldsymbol{\Phi}(t)]
$$

For a multicomponent liquid in $\mathcal{D} \geqslant 2$ spatial dimensions it reads [20]

$$
\begin{aligned}
\mathcal{F}_{k}^{\alpha \beta}[\boldsymbol{X}, \boldsymbol{Y}]= & \frac{\Omega_{\mathcal{D}-1}}{(4 \pi)^{\mathcal{D}}} \sum_{\alpha^{\prime}, \beta^{\prime}, \alpha^{\prime \prime}, \beta^{\prime \prime}} \int_{0}^{\infty} \mathrm{d} p \int_{|k-p|}^{k+p} \mathrm{~d} q V_{k ; p, q}^{\alpha \beta ; \alpha^{\prime} \beta^{\prime}, \alpha^{\prime \prime} \beta^{\prime \prime}} \\
& \times X_{p}^{\alpha^{\prime} \beta^{\prime}} Y_{q}^{\alpha^{\prime \prime} \beta^{\prime \prime}}
\end{aligned}
$$

where the so-called vertices are given by

$$
\begin{gathered}
V_{k ; p, q}^{\alpha \beta ; \alpha^{\prime} \beta^{\prime}, \alpha^{\prime \prime} \beta^{\prime \prime}}=\frac{n}{x_{\alpha} x_{\beta}} \frac{p q}{k^{\mathcal{D}+2}} v_{k p q}^{\alpha \alpha^{\prime} \alpha^{\prime \prime}} v_{k p q}^{\beta \beta^{\prime} \beta^{\prime \prime}}, \\
v_{k p q}^{\alpha \beta \gamma}=\frac{\left(k^{2}+p^{2}-q^{2}\right) c_{p}^{\alpha \beta} \delta_{\alpha \gamma}+\left(k^{2}-p^{2}+q^{2}\right) c_{q}^{\alpha \gamma} \delta_{\alpha \beta}}{\left[4 k^{2} p^{2}-\left(k^{2}+p^{2}-q^{2}\right)^{2}\right]^{(3-\mathcal{D}) / 4}} .
\end{gathered}
$$

$c_{k}^{\alpha \beta}$ denote the direct correlation functions and $\Omega_{\mathcal{D}}=$ $2 \pi^{\mathcal{D} / 2} / \Gamma(\mathcal{D} / 2)$ is the surface of the $\mathcal{D}$-dimensional unit sphere. $\Gamma(x)$ is the Gamma function. $\boldsymbol{c}$ is related to $\boldsymbol{S}$ via the Ornstein-Zernike equation $\left(\boldsymbol{S}^{-1}\right)_{k}^{\alpha \beta}=\delta_{\alpha \beta} / x_{\alpha}-n c_{k}^{\alpha \beta}$.

\section{B. Discretized model}

For practical purposes, we follow Ref. [20] and discretize the wave number $k$ to a finite, equally spaced grid of $K$ points $k=\left(\hat{o}_{d}+\hat{k}\right) \Delta k$ with $\hat{k}=0,1, \ldots, K-1$ and $0<\hat{o}_{d}<1$. The integrals in Eq. (9) are then replaced with Riemann sums

$$
\int_{0}^{\infty} \mathrm{d} p \int_{|k-p|}^{k+p} \mathrm{~d} q \ldots \mapsto(\Delta k)^{2} \sum_{\hat{p}=0}^{K-1} \sum_{\hat{q}=|\hat{k}-\hat{p}|}^{\min \{K-1, \hat{k}+\hat{p}\}} \cdots
$$

and Eq. (7) represents a finite number of coupled nonlinear integrodifferential equations.

The discretized MCT equations shall be applied to the binary hard disk mixture in $2 \mathrm{D}$ introduced in Sec. III. For this, we choose $K=250, \Delta k \cdot d_{b}=0.3$, and $\hat{o}_{2}=0.303$. Static input for the MCT calculations presented in this paper is taken from our BD simulations. For the numerical solution of Eq. (7) we use the algorithm described in Ref. [24]. Our time grids consist of 256 points, and as the initial time step size we choose $2.5 \times 10^{-11}$ units of $d_{b}^{2} / D_{0}$.

\section{Glass transition singularities}

The nonergodicity parameters (NEPs) are the elements of the matrix $\boldsymbol{F}=\lim _{t \rightarrow \infty} \boldsymbol{\Phi}(t)$. For the discretized model above, it can be proved [25] that $\boldsymbol{F}$ is (with respect to $\succeq$ ) the maximum real, symmetric fixed point of the nonlinear map

$$
\mathcal{I}[X]=S-\left(S^{-1}+\mathcal{F}[X, X]\right)^{-1} .
$$

For the binary hard disk model, MCT predicts that the liquid-to-glass transition takes place at a locally smooth critical surface $\mathcal{H}$ that we can represent as the critical packing fraction $\varphi^{c}\left(x_{s}, \delta\right)$ as a function of the concentration of the smaller disks and the size ratio. At this surface $\boldsymbol{F}$ jumps from $\mathbf{0}$ (liquid) to some $\boldsymbol{F}^{c} \succ \mathbf{0}$ (glass). Quantities corresponding to critical points are indicated by a superscript $c$ in the following. General properties of $\mathcal{H}$ have been discussed in detail in Ref. [20].

Besides $\boldsymbol{F}^{c}$, there are further important quantities characterizing a generic liquid-to-glass transition point that is an $A_{2}$ singularity according to the classification of Arnol'd [26]. 
Linearization of $\mathcal{I}^{c}$ around $\boldsymbol{F}^{c}$ yields a so-called positive linear map [25],

$$
\mathcal{C}^{c}[\boldsymbol{Y}]=2\left(\boldsymbol{S}^{c}-\boldsymbol{F}^{c}\right) \mathcal{F}^{c}\left[\boldsymbol{F}^{c}, \boldsymbol{Y}\right]\left(\boldsymbol{S}^{c}-\boldsymbol{F}^{c}\right),
$$

with $\mathcal{C}^{c}[\boldsymbol{Y}] \succeq \mathbf{0}$ for all $\boldsymbol{Y} \succeq \mathbf{0}$. This map has a nondegenerated maximum eigenvalue $r=1$ with a corresponding (right) eigenvector $\boldsymbol{H}^{c}$ and a corresponding left eigenvector $\hat{\boldsymbol{H}}^{c}$ that is an eigenvector to eigenvalue $r^{*}=1$ of the adjoint map of $\mathcal{C}^{c}$ with respect to the scalar product defined in Sec. II A. These two eigenvectors are determined uniquely if we require the normalization

$$
\left(\hat{\boldsymbol{H}}^{c} \mid \boldsymbol{H}^{c}\right)=\left(\hat{\boldsymbol{H}}^{c} \mid \boldsymbol{H}^{c}\left\{\boldsymbol{S}^{c}-\boldsymbol{F}^{c}\right\}^{-1} \boldsymbol{H}^{c}\right)=1 .
$$

A further important quantity is the so-called exponent parameter

$$
\lambda^{c}=\left(\hat{\boldsymbol{H}}^{c} \mid\left\{\boldsymbol{S}^{c}-\boldsymbol{F}^{c}\right\} \mathcal{F}^{c}\left[\boldsymbol{H}^{c}, \boldsymbol{H}^{c}\right]\left\{\boldsymbol{S}^{c}-\boldsymbol{F}^{c}\right\}\right),
$$

whose value determines the exponents in the asymptotic scaling laws (see the next section). These positive exponents are the critical exponent $a$ obeying the relation

$$
\Gamma^{2}(1-a) / \Gamma(1-2 a)=\lambda^{c},
$$

the von Schweidler exponent $b$ satisfying

$$
\Gamma^{2}(1+b) / \Gamma(1+2 b)=\lambda^{c},
$$

and the exponent

$$
\gamma=(a+b) /(2 a b)
$$

describing the divergence of the time scale for the final relaxation of $\boldsymbol{\Phi}(t)$ to $\mathbf{0}$ upon increasing $\varphi$ toward its critical value $\varphi^{c}$.

\section{Asymptotic scaling laws}

Close to the liquid-glass transition, MCT makes universal predictions for the relaxation behavior of $\boldsymbol{\Phi}(t)$ that can be studied in the framework of asymptotic expansions. For the following, let us fix $x_{s}$ and $\delta$ to some specific value and define the distance parameter

$$
\varepsilon=\left(\varphi-\varphi^{c}\right) / \varphi^{c} .
$$

Finally, we introduce the separation parameter $\sigma(\varepsilon)$, which is a linear function of $\varepsilon$. It follows from

$$
\begin{aligned}
\tilde{\sigma}(\varphi)= & \left(\hat{\boldsymbol{H}}^{c} \mid\left\{\boldsymbol{S}^{c}-\boldsymbol{F}^{c}\right\} \boldsymbol{S}^{c-1}\left\{\boldsymbol{S} \mathcal{F}\left[\boldsymbol{F}^{c}, \boldsymbol{F}^{c}\right]\left(\boldsymbol{S}-\boldsymbol{F}^{c}\right)\right.\right. \\
& \left.\left.-\boldsymbol{S}^{c} \mathcal{F}^{c}\left[\boldsymbol{F}^{c}, \boldsymbol{F}^{c}\right]\left(\boldsymbol{S}^{c}-\boldsymbol{F}^{c}\right)\right\}\right)
\end{aligned}
$$

by expanding around $\varphi^{c}$ up to linear order in $\varepsilon[4,27]$.

\section{The first scaling-law regime}

For small separation parameters, $\boldsymbol{\Phi}(t)$ develops powerlaw dynamics located around $\boldsymbol{F}^{c}$, which is also called the $\beta$-relaxation process. For times within the so-called first scaling-law regime defined by $\left|\boldsymbol{\Phi}(t)-\boldsymbol{F}^{c}\right| \ll 1$, also called the $\beta$-scaling regime, there holds the factorization theorem $[4,19]$

$$
\boldsymbol{\Phi}(t)-\boldsymbol{F}^{c}=\boldsymbol{H}^{c} \mathcal{G}(t)+\mathcal{O}(|\sigma|),
$$

with the $\beta$ correlator $\mathcal{G}(t)=\mathcal{O}\left(|\sigma|^{1 / 2}\right)$ obeying the equation of motion

$$
\sigma+\lambda^{c} \mathcal{G}^{2}(t)=\frac{\mathrm{d}}{\mathrm{d} t} \int_{0}^{t} \mathrm{~d} t^{\prime} \mathcal{G}\left(t-t^{\prime}\right) \mathcal{G}\left(t^{\prime}\right)
$$

with the divergent initial condition

$$
\mathcal{G}(t \rightarrow 0)=\left(t / t_{0}\right)^{-a} .
$$

The $\varepsilon$-independent time scale $t_{0}$ has to be matched to the full solution of Eq. (7) at $\varepsilon=0$ since in this case the power law occurring in Eq. (24) is a special solution of Eq. (23) that describes the relaxation of $\boldsymbol{\Phi}(t)$ toward $\boldsymbol{F}^{c}$. It is easy to verify that $\mathcal{G}(t)$ obeys the scaling law

$$
\begin{gathered}
\mathcal{G}(t, \sigma \gtrless 0)=|\sigma|^{1 / 2} \tilde{\mathcal{G}}\left(\tilde{t}=t / t_{\sigma}, \tilde{\sigma}= \pm 1\right), \\
t_{\sigma}=t_{0}|\sigma|^{-\frac{1}{2 a}} .
\end{gathered}
$$

The master function $\tilde{\mathcal{G}}(\tilde{t})$ obeys Eq. (23) with the replacement $\sigma \mapsto \sigma /|\sigma|= \pm 1$ and the initial condition $\tilde{\mathcal{G}}(\tilde{t} \rightarrow 0)=(\tilde{t})^{-a}$. Equation (23) can be solved by asymptotic series expansions [28]. One finds the leading long-time asymptotes

$$
\begin{gathered}
\mathcal{G}(t \rightarrow \infty, \sigma \geqslant 0)=\sqrt{\sigma /\left(1-\lambda^{c}\right)}, \\
\mathcal{G}(t \rightarrow \infty, \sigma<0)=-(t / \tau)^{b} .
\end{gathered}
$$

Equation (27) describes the asymptotic behavior of $\boldsymbol{F}$ in the glassy regime close to $\varphi^{c}$. Equation (28) is referred to as the von Schweidler law and describes the initial part of the relaxation of $\boldsymbol{\Phi}(t)$ from $\boldsymbol{F}^{c}$ to $\mathbf{0}$. Equations (25) and (26) imply

$$
\tau=\tilde{\tau} t_{0}|\sigma|^{-\gamma}
$$

with the $\varepsilon$-independent constant $\tilde{\tau}$, which demonstrates that the arrest of $\boldsymbol{\Phi}(t)$ to $\boldsymbol{F}^{c}$ at $\varphi=\varphi^{c}$ is caused by a power-law divergence of the time scale for the onset of the relaxation of $\boldsymbol{\Phi}(t)$ from $\boldsymbol{F}^{c}$ to $\mathbf{0}$ upon increasing $\varphi$ toward its critical value $\varphi^{c}$.

\section{The second scaling-law regime}

Now we turn to the relaxation process of $\boldsymbol{\Phi}(t)$ from $\boldsymbol{F}^{c}$ to $\mathbf{0}$ within the liquid regime, which is also called the $\alpha$-relaxation process. Considering the limits $\varepsilon \rightarrow 0^{-}$and $t \rightarrow \infty$ with fixed $\tilde{t}=t / \tau$, one arrives at the so-called $\alpha$-scaling law $[4,19]$

$$
\boldsymbol{\Phi}(t)=\tilde{\boldsymbol{\Phi}}^{c}(\tilde{t})+\mathcal{O}(|\sigma|),
$$

where the $\varepsilon$-independent master function $\tilde{\boldsymbol{\Phi}}^{c}(\tilde{t})$ obeys the equation of motion

$$
\left(\boldsymbol{S}^{c}\right)^{-1} \tilde{\boldsymbol{\Phi}}^{c}(\tilde{t})=\tilde{\boldsymbol{m}}^{c}(\tilde{t}) \boldsymbol{S}^{c}-\frac{\mathrm{d}}{\mathrm{d} \tilde{t}} \int_{0}^{\tilde{t}} \mathrm{~d} \tilde{t}^{\prime} \tilde{\boldsymbol{m}}^{c}\left(\tilde{t}-\tilde{t}^{\prime}\right) \tilde{\boldsymbol{\Phi}}^{c}\left(\tilde{t}^{\prime}\right)
$$

with the memory kernel

$$
\tilde{\boldsymbol{m}}^{c}(\tilde{t})=\mathcal{F}^{c}\left[\tilde{\boldsymbol{\Phi}}^{c}(\tilde{t}), \tilde{\boldsymbol{\Phi}}^{c}(\tilde{t})\right]
$$

and the von Schweidler law

$$
\tilde{\boldsymbol{\Phi}}^{c}(\tilde{t} \rightarrow 0)=\boldsymbol{F}^{c}-(\tilde{t})^{b} \boldsymbol{H}^{c}
$$

as the initial condition. Equation (30) is also called the superposition principle due to the following implication: for times within the so-called second scaling-law regime given by $t \gg \tau$, density correlators $\Phi_{k}^{\alpha \beta}(t)$ corresponding to different 
values of $\varepsilon$ collapse onto master curves $\left(\tilde{\boldsymbol{\Phi}}^{c}\right)_{k}^{\alpha \beta}(\tilde{t})$ when they are plotted as functions of $\tilde{t}=t / \tau$.

\section{E. Mixing effects}

As already briefly mentioned in Sec. I, MCT predicts the existence of four mixing effects for binary hard disks in 2D [20]. Let us recapitulate these effects in more detail.

(i) For small size disparities the glassy regime is enhanced. For $0.65 \lesssim \delta<1$ it is $\varphi^{c}\left(x_{s}, \delta\right)<\varphi_{0}^{c}$ if $0<x_{s}<1$, where $\varphi_{0}^{c}$ denotes the critical packing fraction for monodisperse hard disks in 2D. For $\delta=5 / 7$, for instance, $\varphi^{c}\left(x_{s}\right)$ develops a single minimum, located at $x_{s} \approx 0.5$.

(ii) For larger size disparities the liquid state is stabilized; that is, for $\delta \lesssim 0.37$ it is $\varphi^{c}\left(x_{s}, \delta\right)>\varphi_{0}^{c}$ if $0<x_{s}<1$. This effect is also called plasticization. For $\delta=1 / 3$, for instance, $\varphi^{c}\left(x_{s}\right)$ develops a single maximum, located at $x_{s} \approx 0.85$.

(iii) Upon increasing the concentration $x_{s}$ of the smaller particles, the NEPs, and thus also the plateau values of the normalized correlation functions $\Phi_{k}^{\alpha \alpha}(t) / S_{k}^{\alpha \alpha}$ in the liquid regime for intermediate times, increase for $k$ not too small and all $x_{s}$.

(iv) Starting with $x_{s}=0$ and increasing the concentration $x_{s}$ of the smaller particles leads, for not too large $k$, to a slowing-down of the relaxation of the normalized correlators $\Phi_{k}^{b b}(t) / S_{k}^{b b}$ of the big particles toward their plateaus, in the sense that the $\Phi_{k}^{b b}(t) / S_{k}^{b b}$ versus $\log _{10}(t)$ curve becomes flatter upon increasing $x_{s}$.

The amplitude of predicted effects (i) and (ii) is quite low; the total variation of $\varphi^{c}\left(x_{s}, \delta\right)$ is of the order of $1 \%$. Nevertheless, these small changes in $\varphi^{c}$ may have a strong influence on time-dependent quantities that are accessible to our BD simulations. Let us fix some $\delta$ and $\varphi$ such that for all $0<x_{s}<1$, the condition $0<\left[\varphi^{c}\left(x_{s}, \delta\right)-\varphi\right] \ll 1$ is satisfied. Equations (29) and (30) predict, then, a strong variation in the time scales $\tau$ for the $\alpha$ relaxation of the correlators $\Phi_{k}^{\alpha \beta}(t)$. It has been demonstrated [20] that an occurring minimum (maximum) in $\varphi^{c}\left(x_{s}\right)$ is then directly reflected by an occurring maximum(minimum) in $\tau$. Using this information, we demonstrate in the following that the mixing effects (i-iv) predicted by MCT are indeed observable in our BD simulation data.

\section{RESULTS AND DISCUSSION}

We choose to use a BD simulation for our comparison with MCT results for the following reason: the behavior of the relaxation times of the system upon approaching the glass transition point, the details of the $\alpha$ relaxation, and the NEPs are independent of the microscopic dynamics of supercooled liquids, as Gleim et al. showed in Ref. [29]. Nevertheless, the relaxation onto the plateaus is expected to be described better within MCT for BD [29].

\section{A. Statics}

The first point we want to address are the NEPs. A common description of $\alpha$ relaxation is in terms of stretched exponential Kohlrausch laws,

$$
\Phi_{k}^{\alpha \alpha}(t)=A_{k}^{\alpha \alpha} \exp \left[-\left(t / \tilde{\tau}_{k}^{\alpha \alpha}\right)^{\beta_{k}^{\alpha \alpha}}\right],
$$

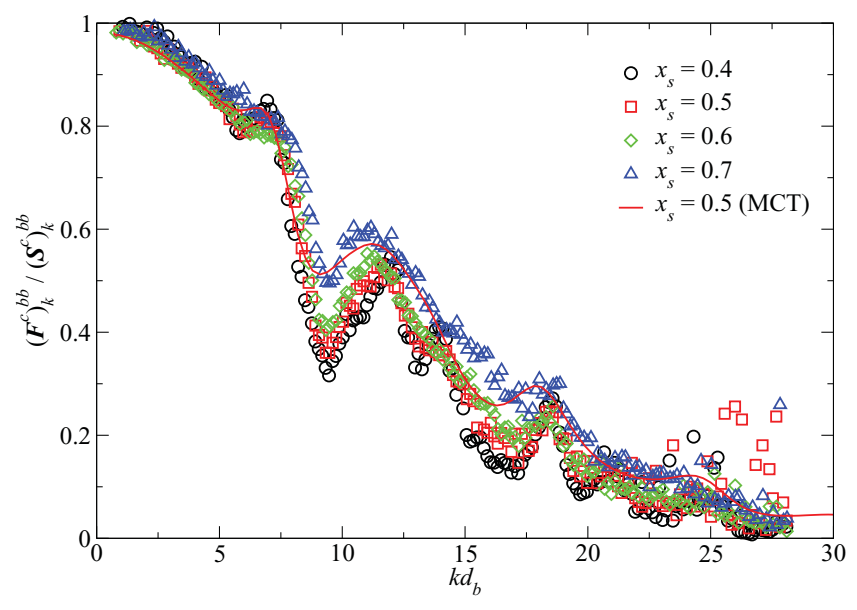

FIG. 1. (Color online) Normalized critical nonergodicity parameters of the simulated collective density correlators $\Phi_{k}^{b b}(t)$ of big particles, extracted from Kohlrausch fits for size ratio $\delta=5 / 7$ and packing fraction $\varphi=0.79$. Number concentrations of small particles $x_{s}$ vary as labeled in the key. The solid (red) line gives the MCT results calculated with a simulated structure factor input at $\varphi_{\mathrm{MCT}}^{c}=0.6920$ and $x_{s}=0.5$.

with stretching exponent $\beta_{k}^{\alpha \alpha}$, relaxation time scale $\tilde{\tau}_{k}^{\alpha \alpha}$, and amplitude $A_{k}^{\alpha \alpha}$. For structural relaxation in equilibrium system $\beta_{k}^{\alpha \alpha}<1$ is required. The $\alpha$ master function from MCT, Eq. (31), is different from the Kohlrausch form; however, the theory predicts that for large wave numbers the two functional forms become identical and $\beta_{k}^{\alpha \alpha} \rightarrow b$ [30]. The Kohlrausch amplitude $A_{k}^{\alpha \alpha}$ provides an estimate for the MCT NEPs $\left(\boldsymbol{F}^{c}\right)_{k}^{\alpha \alpha}$. Since the $\alpha$ process starts below this plateau value, $A_{k}^{\alpha \alpha} \leqslant\left(\boldsymbol{F}^{c}\right)_{k}^{\alpha \alpha}$ should hold. However, in practice, the separation of the $\alpha$ process from the $\beta$ relaxation is not clear enough to fulfill this prediction.

Kohlrausch fits are hindered by some subtle problems: lacking a clear separation of the $\alpha$ process, the fit parameters enclose a dependence on the fit range. A priori it is unclear how to choose the optimal fit range, as for very long times, one expects the relaxation to become (nonstretched) exponential again, and for short times, deviations stemming from $\beta$ relaxation hamper the choice. The fit range was fixed, so that the parameters only exhibit the weakest (the region where they are almost constant) dependence on the boundaries. This procedure leads to $t \in[7.65,2551.02]$ for $\delta=5 / 7$ and $\varphi=0.79$ with various $x_{s}$. For $\delta=1 / 3$ and $\varphi=0.81$ we used $t \in[1.39,555.56]$ for the various $x_{s}$.

Figure 1 shows approximate values for the normalized critical NEPs $\left(\boldsymbol{F}^{c}\right)_{k}^{b b} /\left(\boldsymbol{S}^{c}\right)_{k}^{b b}$ for big disks at $\delta=5 / 7$ and different values of $x_{s}$, extracted from our BD simulation data via Kohlrausch fits. Corresponding results for smaller disks are shown in Fig. 2. In both Fig. 1 and Fig. 2, we have also included critical NEPs for $x_{s}=0.5$, calculated via MCT with BD-simulated structure factors as input, with MCT yielding a critical packing fraction of $\varphi_{\mathrm{MCT}}^{c}=0.6920$ for the simulated input. On a qualitative level, our MCT results are in good agreement with our BD simulation results. For big particles the relation $A_{k}^{\alpha \alpha} \leqslant\left(\boldsymbol{F}^{c}\right)_{k}^{\alpha \alpha}$ is well fulfilled for all $k d_{b}$ except for some outliers. The same holds for small particles, but for $k d_{b} \lesssim 5$ the Kohlrausch fit yields smaller estimations for 


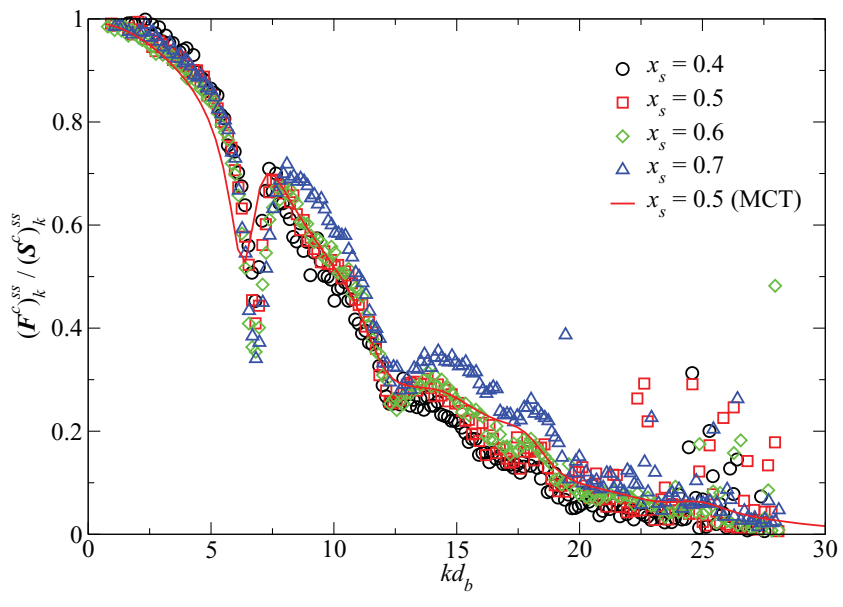

FIG. 2. (Color online) Normalized critical nonergodicity parameters of the simulated collective density correlators $\Phi_{k}^{s s}(t)$ of the small particles, extracted from Kohlrausch fits for the size ratio $\delta=5 / 7$ and the packing fraction $\varphi=0.79$. Number concentrations of small particles $x_{s}$ are the same as in Fig. 1. The solid (red) line shows the MCT results calculated with simulated structure factors as in Fig. 1.

NEPs. In both Fig. 1 and Fig. 2, we observe a slight increase in the BD simulation results for NEPs upon increasing $x_{s}$, which, on a qualitative level, is in agreement with previous MCT results [6,20].

Figure 3 shows approximate values for the normalized critical NEPs $\left(\boldsymbol{F}^{c}\right)_{k}^{b b} /\left(\boldsymbol{S}^{c}\right)_{k}^{b b}$ for big disks at $\delta=1 / 3$ and different values of $x_{s}$, extracted from our BD simulation data via Kohlrausch fits. Corresponding results for the NEPs of smaller disks are shown in Fig. 4. In both Fig. 3 and Fig. 4, we have also included critical NEPs for $x_{s}=0.5$, calculated via MCT with BD-simulated structure factors as input, giving a critical packing fraction of $\varphi_{\mathrm{MCT}}^{c}=0.6991$ for the simulated input. For the present value of $\delta$, we observe that our MCT calculations yield systematically smaller values for NEPs,

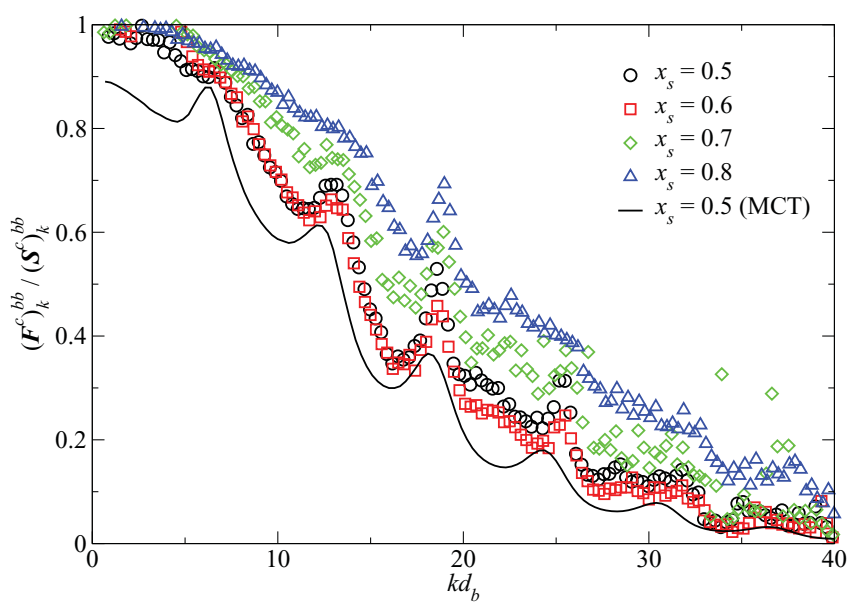

FIG. 3. (Color online) Normalized critical nonergodicity parameters of simulated collective density correlators $\Phi_{k}^{b b}(t)$ of big particles, extracted from Kohlrausch fits for size ratio $\delta=1 / 3$ and packing fraction $\varphi=0.81$. The various number concentrations $x_{s}$ are as shown in the key. MCT results using simulated structure factors as input with $\varphi_{\mathrm{MCT}}^{c}=0.6991$ are shown as the solid (black) line for $x_{s}=0.5$.

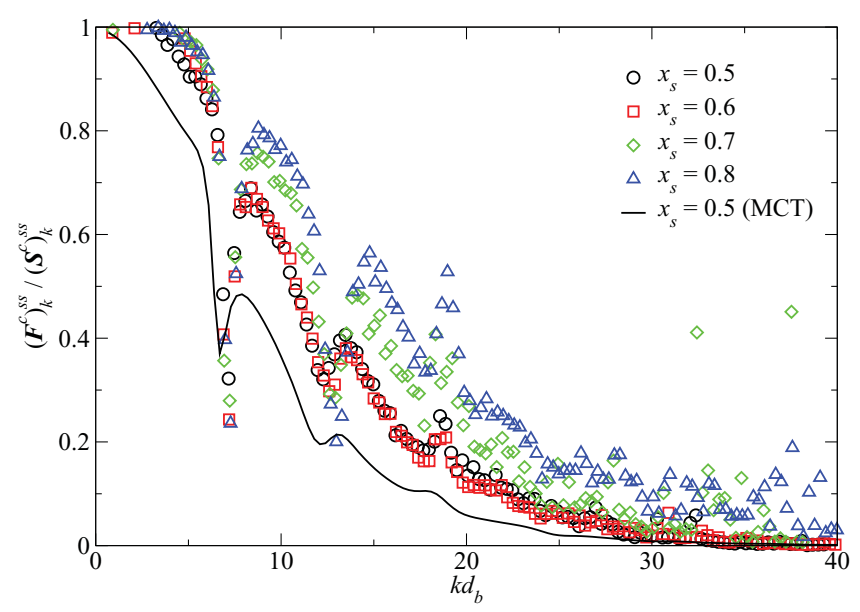

FIG. 4. (Color online) Normalized critical nonergodicity parameters of the simulated collective density correlators $\Phi_{k}^{s s}(t)$ of small particles, extracted from Kohlrausch fits for size ratio $\delta=1 / 3$ and packing fraction $\varphi=0.81$. The color and symbol coding for the different $x_{s}$ is the same as in Fig. 3. The solid (black) line shows MCT results calculated with simulated structure factors as used in Fig. 3.

compared to our BD simulation results. The underestimation of the NEPs may be attributed to the underestimation of $\varphi^{c}$ : MCT predicts arrest at lower densities, but the NEPs may increase with density, as the denser glass is stiffer with respect to density fluctuations. The BD simulation results in both Fig. 3 and Fig. 4 indicate a systematic increase in NEPs upon increasing $x_{s}$ that is, as expected, more strongly pronounced than for the case $\delta=5 / 7$, which is, on a qualitative level, in agreement with previous MCT results [6,20].

We can conclude here that, at least on a qualitative level, our BD simulation results confirm the $x_{s}$ and $\delta$ dependences of the NEPs predicted by MCT $[6,20]$. In particular, we have clearly verified the existence of mixing effect (iii).

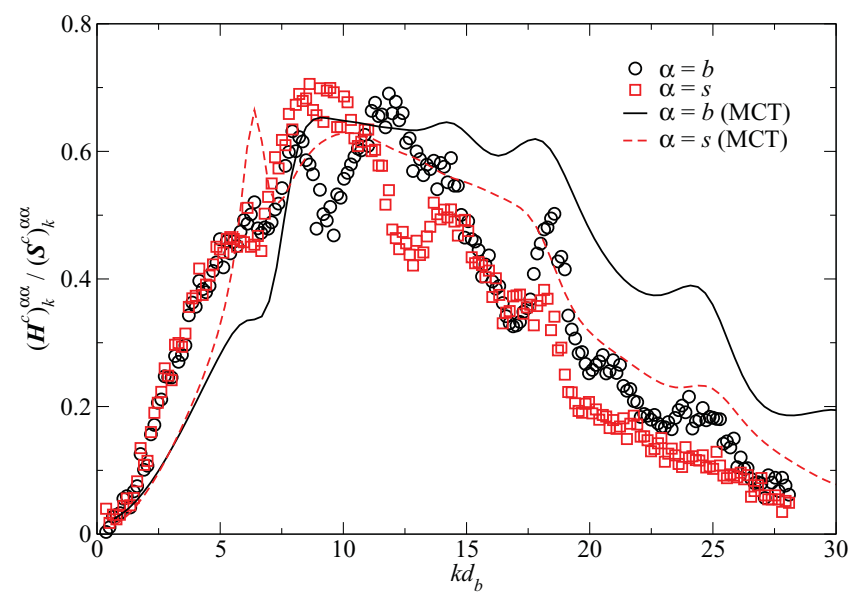

FIG. 5. (Color online) Critical amplitudes obtained with Eq. (35) for big and small particles with $k_{0} d_{b}=2.5$. Data were extracted from the collective correlators at $\varphi=0.79, \delta=5 / 7$, and $x_{s}=0.5$. Solid (black) and dashed (red) lines depict MCT results obtained with the same simulated structure factors as in Figs. 1 and 2. 


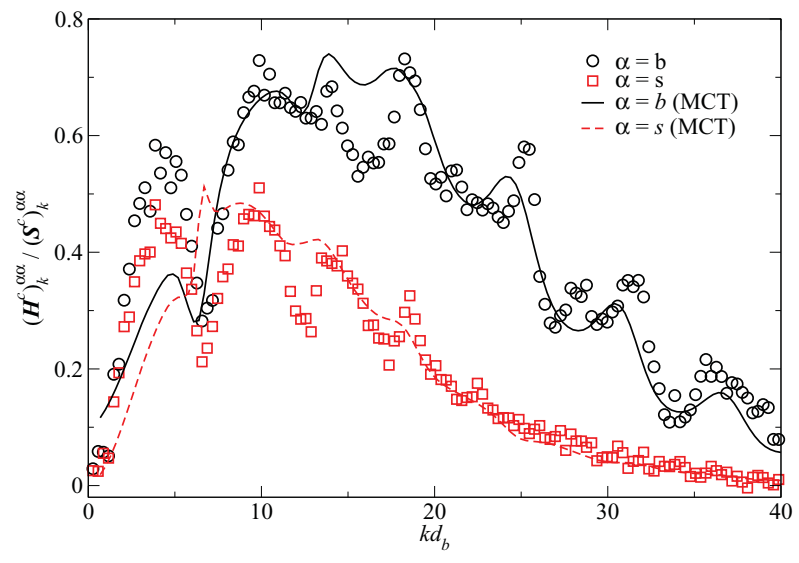

FIG. 6. (Color online) Critical amplitudes obtained by Eq. (35) for $\delta=1 / 3$ and $x_{s}=0.5$ with $k_{0} d_{b}=5.37$ from the simulated correlators at $\varphi=0.81, \delta=1 / 3$, and $x_{s}=0.5$. Solid (black) and dashed (red) lines depict MCT results obtained with the same simulated structure factors as used in Figs. 3 and 4.

Let us investigate the so-called critical amplitude as a further interesting static quantity. Figure 5 shows approximate values for the normalized critical amplitudes $\left(\boldsymbol{H}^{c}\right)_{k}^{\alpha \alpha} /\left(\boldsymbol{S}^{c}\right)_{k}^{\alpha \alpha}$ for both big and small disks at $\delta=5 / 7$ and $x_{s}=0.5$, extracted from our BD simulations. Corresponding results for $\delta=1 / 3$ are shown in Fig. 6. To determine the critical amplitudes from the BD simulation data, one can define the function [31]

$$
Y_{k}^{\alpha \beta}=\frac{\sum_{j=1}^{n / 2} \Phi_{k}^{\alpha \beta}\left(t_{j}\right)-\sum_{j=n / 2+1}^{n} \Phi_{k}^{\alpha \beta}\left(t_{j}\right)}{\sum_{j=1}^{n / 2} \Phi_{k_{0}}^{\alpha \beta}\left(t_{j}\right)-\sum_{j=n / 2+1}^{n} \Phi_{k_{0}}^{\alpha \beta}\left(t_{j}\right)}=\frac{\left(\boldsymbol{H}^{c}\right)_{k}^{\alpha \beta}}{\left(\boldsymbol{H}^{c}\right)_{k_{0}}^{\alpha \beta}},
$$

with $t_{j}$ chosen in the $\beta$-scaling regime, which, in our case, is determined to $t_{j} \in[0.47449,8.2882]$ for $\delta=5 / 7$ and $t_{j} \in$ $[0.10334,1.8051]$ for $\delta=1 / 3$. The last equality follows from Eq. (22) and thus allows us to extract the critical amplitudes $\left(\boldsymbol{H}^{c}\right)_{k}^{\alpha \beta}$ up to a factor $\left(\boldsymbol{H}^{c}\right)_{k_{0}}^{\alpha \beta}$. Besides the numerical uncertainty at low $k d_{b}$, the simulation data for $\delta=1 / 3$ are in good agreement with our corresponding results from MCT using BD-simulated structure factors as input. For the case $\delta=5 / 7$ we observe larger discrepancies for all $k d_{b}$.

\section{B. Dynamics}

In this section the most important quantities for MCT, the collective density correlators, are discussed. Figure 7 shows normalized collective density correlators $\Phi_{k}^{b b}(t) / S_{k}^{b b}$ from our BD simulations for big particles of binary hard disk mixtures in $2 \mathrm{D}$ at $\delta=5 / 7, \varphi=0.79$, and $k d_{b}=8.5$ for different concentrations $x_{s}$ of smaller disks. Similar results for $\Phi_{k}^{s s}(t) / S_{k}^{s s}$ are shown in Fig. 8. Focusing on the data for $\Phi_{k}^{b b}(t) / S_{k}^{b b}$ in Fig. 7, these data exhibit the same three mixing effects as the data from MCT shown in Fig. 6 in Ref. [20], namely, (iii) an increase in the plateau values, accompanied by (iv) a slowing-down of the relaxation toward these plateaus and (i) an additional slowing-down of the $\alpha$-relaxation process upon increasing $x_{s}$. However, there are some deviations: from Fig. 5 in Ref. [20] we would expect that the slowest $\alpha$-relaxation process occurs at $x_{s} \cong 0.5$. Simulation data, however, exhibit the slowest $\alpha$ relaxation at the highest investigated value, $x_{s}=0.7$. Unfortunately, the

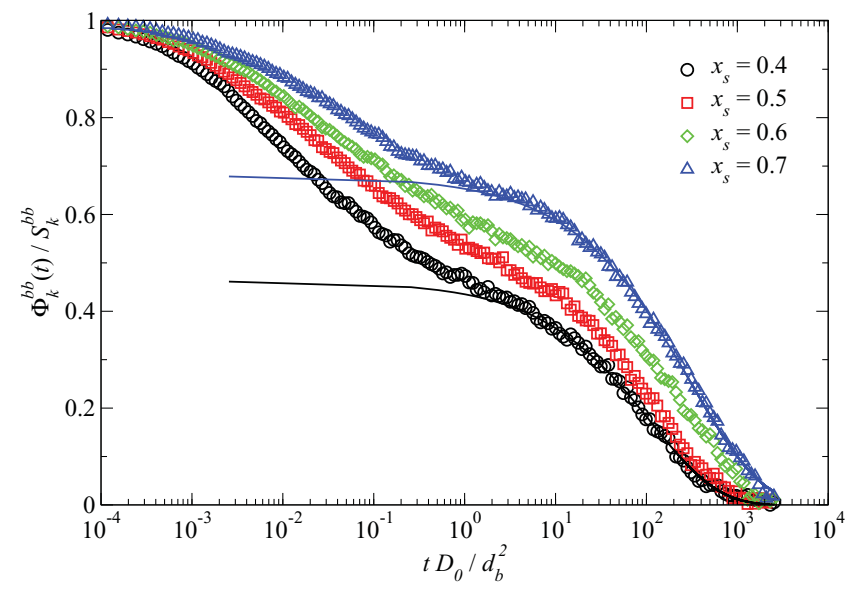

FIG. 7. (Color online) Simulated normalized collective correlation functions of big particles for size ratio $\delta=5 / 7$ at $\varphi=0.79$ and $k d_{b}=8.5$ for varying $x_{s} \in\{0.4,0.5,0.6,0.7\}$ as labeled in the key. Solid lines show examples of Kohlrausch fits to the $x_{s}=0.4$ correlator (black) and the $x_{s}=0.7$ correlator (blue).

simulation systems at higher $x_{s}$ are subject to crystallization, which makes them unsuitable for the MCT comparison, rendering this region "unaccessible" for simulations. A possible source of the discrepancy is that in Ref. [20], an approximate theory, the Percus-Yevick structure factor, was used as input for the MCT calculations. Furthermore, for $\delta=5 / 7$, MCT predicts $0.686<\varphi^{c}<0.6920$ for all $x_{s}$. The simulation data, however, imply $\varphi_{\text {sim }}^{c} \gtrsim 0.79$, which means that MCT underestimates the critical packing fraction by about $15 \%$. The Percus-Yevick approximation contributes to this underestimation, but even with simulated structure factors as input, MCT underestimates the critical packing fraction. In Fig. 8 we basically find the same scenario as in Fig. 7, although a bit less pronounced, as expected from MCT [32].

Figure 9 shows the normalized collective density correlators $\Phi_{k}^{b b}(t) / S_{k}^{b b}$ from our BD simulations at $\delta=1 / 3, \varphi=0.81$, and $k d_{b}=9.0$ for different concentrations $x_{s}$ of smaller disks.

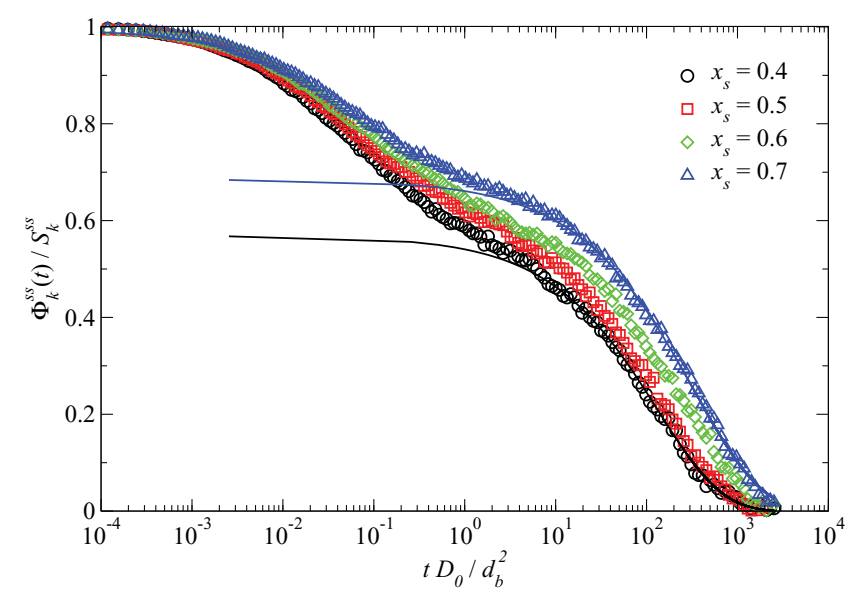

FIG. 8. (Color online) Simulated normalized collective correlation functions of small particles for size ratio $\delta=5 / 7$ at $\varphi=0.79$ and $k d_{b}=8.5$ for varying $x_{s}$. Color and symbol coding is the same as in Fig. 7. Solid lines show examples of Kohlrausch fits to the $x_{s}=0.4$ correlator (black) and the $x_{s}=0.7$ correlator (blue). 


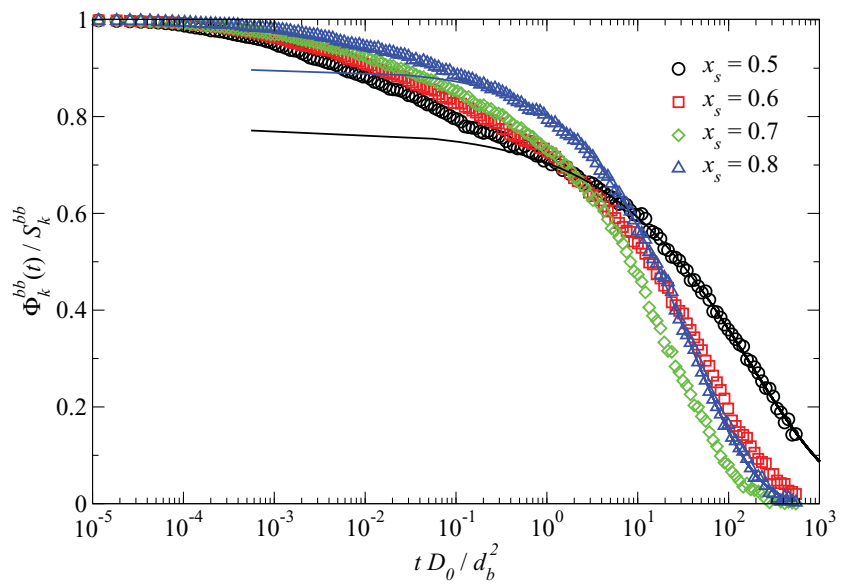

FIG. 9. (Color online) Simulated normalized collective correlation functions of big particles for size ratio $\delta=1 / 3$ at $\varphi=0.81$ and $k d_{b}=9.0$ for varying $x_{s} \in\{0.5,0.6,0.7,0.8\}$ as labeled in the key. Solid lines show exemplary Kohlrausch fits to the $x_{s}=0.5$ correlator (black) and the $x_{s}=0.8$ correlator (blue).

Similar results for $\Phi_{k}^{s s}(t) / S_{k}^{s s}$ are shown in Fig. 10. First, we show a closer look at the data for $\Phi_{k}^{b b}(t) / S_{k}^{b b}$ in Fig. 9. On a qualitative level, the data for the three lowest values of $x_{s}$ are fully consistent with all MCT results in both Fig. 5 in Ref. [20] and Fig. 7 in Ref. [20]. Upon increasing $x_{s}$, the simulation data exhibit mixing effect (iii), an increase in the plateau values, accompanied by (iv) a slowing-down of the relaxation toward these plateaus. In addition, increasing $x_{s}$ from 0.5 to 0.7 leads to (ii) a speeding-up of the $\alpha$ relaxation. Thus, the three correlators corresponding to the lowest values of $x_{s}$ exhibit a pairwise crossing. A further increase in $x_{s}$ to 0.8 leads, again, to a slowing-down of the $\alpha$-relaxation process, although from the MCT results in Fig. 5 in Ref. [20], we would expect the fastest $\alpha$ relaxation at $x_{s} \cong 0.85$. For small particles we observe similar, albeit less pronounced, effects (see Fig. 10).

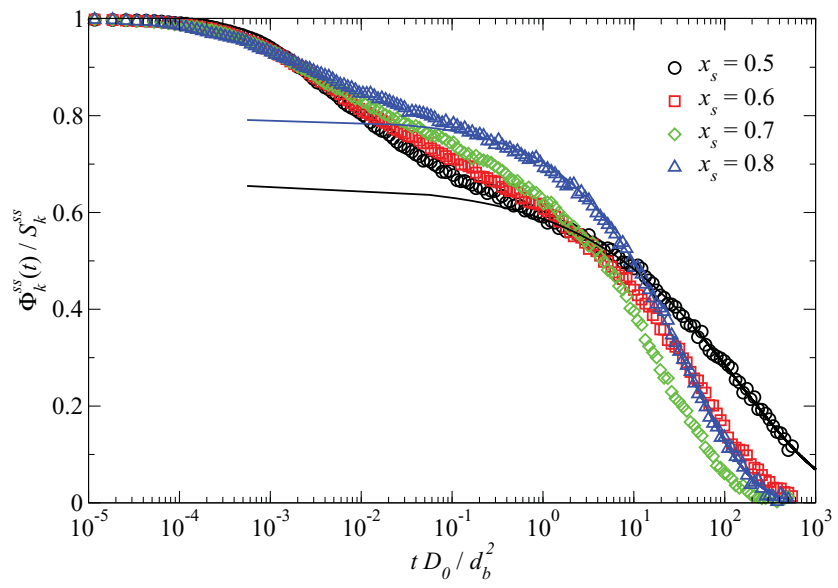

FIG. 10. (Color online) Simulated normalized collective correlation functions of small particles for size ratio $\delta=1 / 3$ at $\varphi=0.81$ and $k d_{b}=9.0$ for varying $x_{s}$. Color and symbol coding is the same as in Fig. 9. Solid lines show exemplary Kohlrausch fits to the $x_{s}=0.5$ correlator (black) and the $x_{s}=0.8$ correlator (blue).
The fact that the simulation data at $\delta=5 / 7$ and $\varphi=0.79$ and those at $\delta=1 / 3$ and $\varphi=0.81$ show very similar $\alpha$-relaxation times is, on a qualitative level, consistent with the MCT result $\varphi^{c}\left(x_{s}, \delta=5 / 7\right)<\varphi^{c}\left(x_{s}, \delta=1 / 3\right)$ for $0<x_{s}<1$. Let us conclude here with the statement that, at least on a qualitative level, the four mixing effects predicted by MCT for the binary hard disk model in 2D [20] are also observable in our computer simulations, which supports the quality of MCT in $2 \mathrm{D}$.

\section{GLASS TRANSITION OF A SELECTED MIXTURE}

In this section we select one of the systems $(\delta=5 / 7$, $x_{s}=0.5$ ) from the preceding sections and perform a more profound MCT analysis including asymptotic checks and determination of the MCT glass transition point. This will complete the MCT analysis of the system already discussed under shear in Ref. [23] with respect to the quiescent state.

In analogy to Eq. (35) it is possible to test another prediction of MCT. To investigate the factorization theorem given by Eq. (22), we consider the function [33]

$$
X_{k}^{\alpha \beta}(t)=\frac{\Phi_{k}^{\alpha \beta}(t)-\Phi_{k}^{\alpha \beta}\left(t^{\prime}\right)}{\Phi_{k}^{\alpha \beta}\left(t^{\prime}\right)-\Phi_{k}^{\alpha \beta}\left(t^{\prime \prime}\right)},
$$

with fixed times $t^{\prime}<t^{\prime \prime}$ to be chosen appropriately from the $\beta$-scaling regime. Then Eq. (22) predicts

$$
X_{k}^{\alpha \beta}(t)=\frac{G(t)-G\left(t^{\prime}\right)}{G\left(t^{\prime}\right)-G\left(t^{\prime \prime}\right)}+\mathcal{O}(|\sigma|)
$$

not to be dependent on wave number and particle index, to leading order in the separation parameter $\sigma$. Thus it must be possible to fix two times, $t^{\prime}$ and $t^{\prime \prime}$, uniquely so that superimposing $X_{k}^{\alpha \beta}(t)$ for different $k$ yields a window in which all $X_{k}^{\alpha \beta}(t)$ collapse. An advantage of this procedure is that the critical amplitude drops out and does not need to be fitted.

Figure 11 shows our BD simulation results for $X_{k}^{\alpha \alpha}(t)$ at $\varphi=0.79, \delta=5 / 7, x_{s}=0.5, \quad t^{\prime} D_{0} / d_{b}^{2}=0.7648$, and $t^{\prime \prime} D_{0} / d_{b}^{2}=9.117$ for different wave numbers. Indeed, within the numerical accuracy of our simulations, for both $\alpha=b$

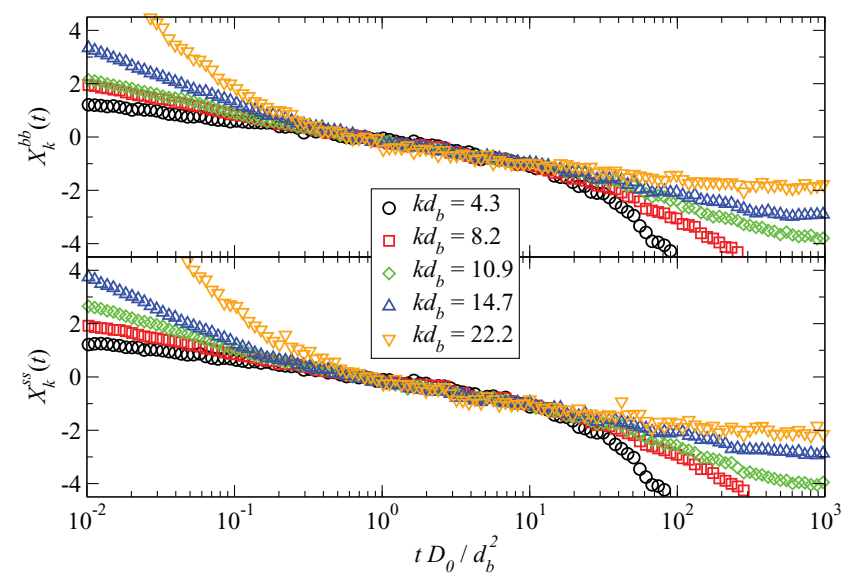

FIG. 11. (Color online) Functions $X_{k}^{\alpha \alpha}(t)$ calculated from Eq. (36) with the simulated correlators at $\varphi=0.79, \delta=5 / 7$, and $x_{s}=0.5$ by fixing $t^{\prime} D_{0} / d_{b}^{2}=0.7648$ and $t^{\prime \prime} D_{0} / d_{b}^{2}=9.117$ for big and small particles. 
and $\alpha=s$ the data for different $k d_{b}$ collapse onto each other within a time window of about two decades, similar to previous results for binary Lennard-Jones mixtures in 3D [33]. A more sensitive test of MCT asymptotics is the so-called ordering rule. Because in the next-to-leading order corrections to the factorization theorem the same $k$-dependent correction amplitudes appear, the deviations before the collapse regime must be in the same direction as after the collapse window. Hence correlators entering the collapse region in a certain order when numbered from top to bottom should leave the collapse window in exactly that ordering [19]. Figure 11 confirms that prediction.

Now we test the validity of the $\alpha$-scaling law given by Eq. (30). According to that equation, plotting the correlators as a function of $t / \tau$ makes the data collapse for long times on a master curve upon approaching $\varphi \rightarrow \varphi^{c}$ from the liquid. To determine a dimensionless relaxation time $\tau / \tau^{*} \propto|\sigma|^{-\gamma}$ from the simulation, the correlators at $k d_{b} \approx 8.5$ (at the structure factor peak of $\sum_{\alpha \beta} S_{k}^{\alpha \beta}$ ) corresponding to $\varphi<0.79$ have been shifted along the $\log _{10}(t)$ axis to coincide in the final decay with the one at the highest packing fraction $\varphi=0.79$. The highest packing fraction defines $\tau_{\varphi=0.79} / \tau^{*}=1$ and yields our best approximation for the $\alpha$ master function. Checking that $\tau / \tau^{*}$ is independent of $k d_{b}$, we can validate the $\alpha$ scaling. We have chosen the structure factor peak for the determination of $\tau / \tau^{*}$, as the strength of the $\alpha$ process is maximal here, and thus a separation from the $\beta$ process can be achieved. Figure 12 shows the exemplary result of the $\alpha$ scaling for two wave numbers. The data clearly exhibit the two-step relaxation pattern of glass-forming liquids with increasingly stretched plateaus upon an increase in the packing fraction. The shifted correlators approach an $\alpha$ master curve, with the highest densities collapsing over almost three decades in time.

After having checked a few asymptotic results, we now present full numerical MCT calculations. As a preliminary we use BD-simulated static structure factors as input for MCT to calculate the critical packing fraction $\varphi_{\mathrm{MCT}}^{c} \cong 0.6920$ and the exponent $\gamma=2.4969$. The obvious mismatch in $\varphi^{c}$ necessitates a comparison, at a corresponding separation from

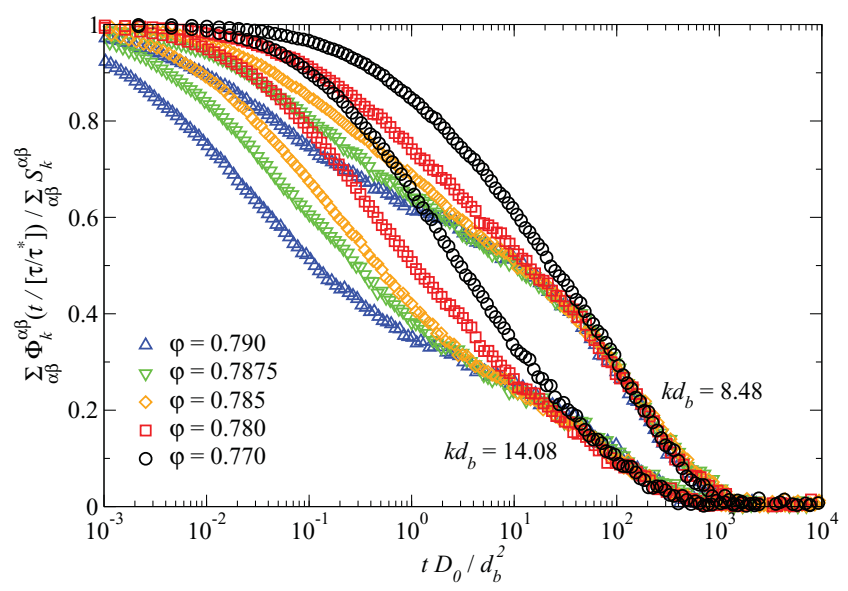

FIG. 12. (Color online) Rescaled correlators for $\varphi \leqslant 0.79, \delta=$ $5 / 7$, and $x_{s}=0.5$ to collapse on one $\alpha$ master function at long times. Rescale times are independent of $k d_{b}$.

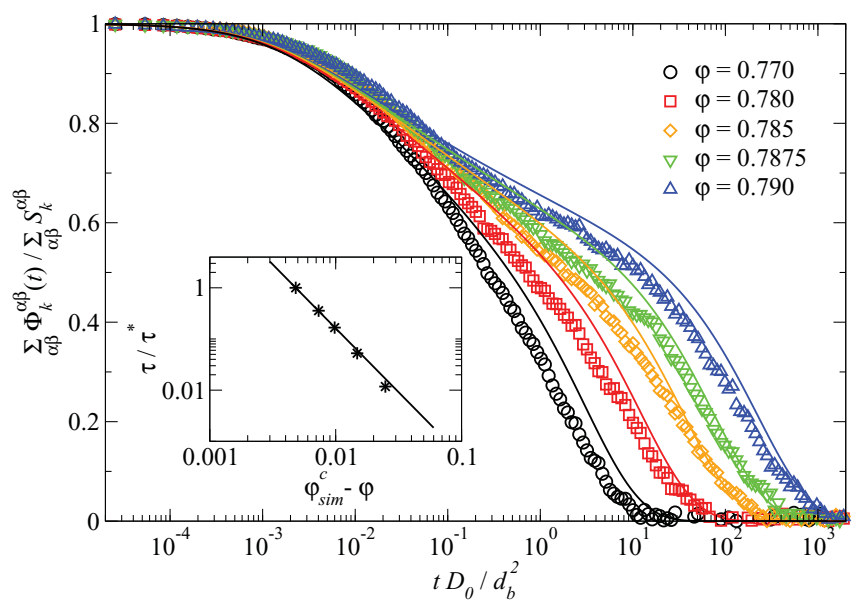

FIG. 13. (Color online) Normalized total collective correlators from BD simulations for $\delta=5 / 7, x_{s}=0.5$, and $k d_{b}=8.5$. Solid lines show corresponding MCT results, where the packing fractions are mapped according to Eq. (38). Inset: $\alpha$-relaxation time scales and the fitted power law $\tau / \tau^{*}=A\left|\varphi-\varphi_{\text {sim }}^{c}\right|^{-\gamma}$, with $\gamma=2.4969$ (fixed from MCT), $A=1.633 \times 10^{-6}$, and $\varphi_{\text {sim }}^{c}=0.7948$. See text for details.

the transition point. This entails matching the separation parameter $\sigma$, which is not easy to obtain from the simulation, but a peculiarity of our system helps us to circumvent the problem. In Fig. 13 we show BD simulation results for the normalized total collective correlators $\sum_{\alpha \beta} \Phi_{k}^{\alpha \beta}(t) / \sum_{\alpha \beta} S_{k}^{\alpha \beta}$ at $\delta=5 / 7, x_{s}=0.5$, and $k d_{b}=8.5$ for different packing fractions $\varphi$ within the liquid regime close to vitrification. The inset in Fig. 13 shows our results for $\tau / \tau^{*}$ obtained from the shifting process shown in Fig. 12. The straight line shows the result from a power-law fit $\tau \sim\left|\varphi-\varphi_{\text {sim }}^{c}\right|^{-\gamma}$ with fixed $\gamma=2.4969$, which yields the extrapolated value $\varphi_{\text {sim }}^{c} \cong 0.79481$ for the critical packing fraction, which is approximately $15 \%$ larger than the value predicted by MCT. For $\left|\varphi-\varphi_{\text {sim }}^{c}\right| \lesssim 0.01$, the $\varphi$ dependence of $\tau / \tau^{*}$ is excellently described by the MCT exponent $\gamma$. We can conclude that although MCT underestimates the critical packing fraction, it nevertheless describes very well the $\varphi$ dependence of the $\alpha$-relaxation process in the liquid regime close to the glass transition, which, again, supports the quality of MCT in 2D.

Using this information, we are able to present a quantitative comparison of time-dependent correlation functions from MCT with those from our BD simulations. For this purpose we have to take into account that MCT overestimates glass formation. It is well known that this results in predicting the glass transition at a critical packing fraction $\varphi^{c}$ that is too low. Hence the relevant parameter when comparing MCT and simulation results is the separation parameter $\sigma$, which depends linearly on the distance parameter via $\sigma=C \varepsilon$ (see Sec. V D). As the constant $C$ is evaluated at the critical packing fraction with the corresponding structure factor, it is reasonable to assume that MCT does not yield the same prefactor as the simulation. For instance, Flenner and Szamel found that both prefactors differ in a 3D binary Lennard-Jones mixture [34]. To construct a mapping of the packing fractions $\varphi_{\text {sim }}$ used in our BD simulation onto some appropriate ones, $\varphi_{\mathrm{MCT}}$ to be 


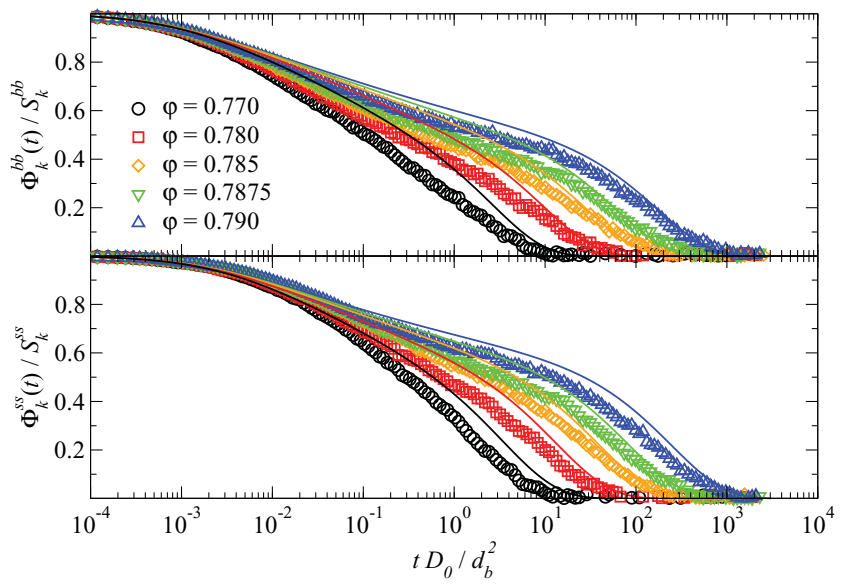

FIG. 14. (Color online) Normalized collective correlators from BD simulations for $\delta=5 / 7, x_{s}=0.5$, and $k d_{b}=8.5$. Solid lines show corresponding MCT results where the packing fractions have been mapped according to Eq. (38).

used for the corresponding MCT calculations, we postulate that the separation parameters for both systems must be equal. This leads us to the ansatz

$$
\varepsilon_{\mathrm{MCT}}=\left(C_{\text {sim }} / C_{\mathrm{MCT}}\right) \varepsilon_{\text {sim }} \equiv A \varepsilon_{\text {sim }}
$$

with some appropriately chosen constant $A$, which, in our special case, can be found empirically as $A \cong 1$. With this, all input parameters for our MCT equations are uniquely determined. The solid lines in Fig. 13 represent our MCT results for the normalized total collective correlation functions corresponding to the BD simulation data shown in Fig. 13. Corresponding results for the normalized partial correlators $\Phi_{k}^{\alpha \alpha}(t) / S_{k}^{\alpha \alpha}$ are shown in Fig. 14. We observe that for the chosen wave number, MCT tends to underestimate the correlation functions in the transient time regime $t D_{0} / d_{b}^{2} \cong 2.5 \times 10^{-3}$ and overestimates the plateau values at times within the $\beta$-scaling regime. Besides these quantitative deviations, MCT describes very well the qualitative $t$ and $\varphi$ dependences of

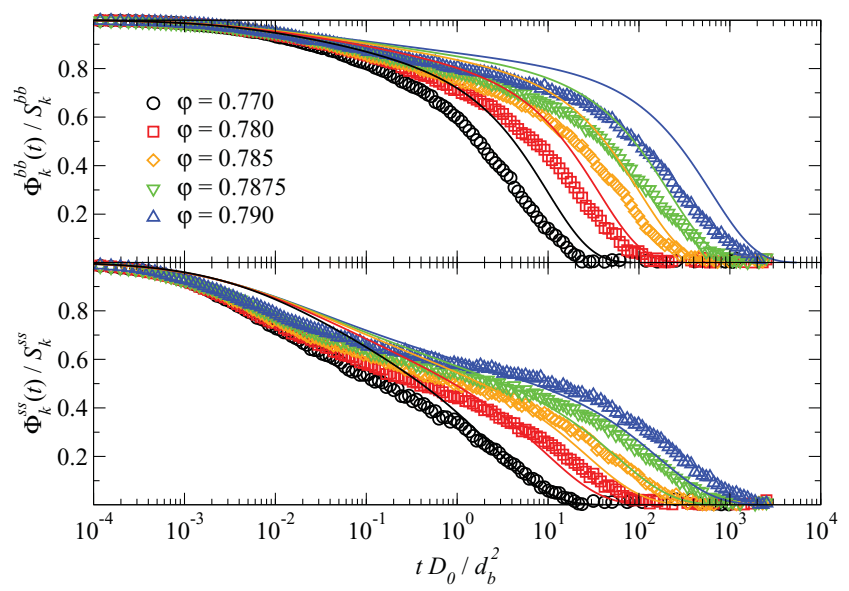

FIG. 15. (Color online) Normalized collective correlators from BD simulations for $\delta=5 / 7, x_{s}=0.5$, and $k d_{b}=6.4$. Solid lines show corresponding MCT results where the packing fractions have been mapped according to Eq. (38).

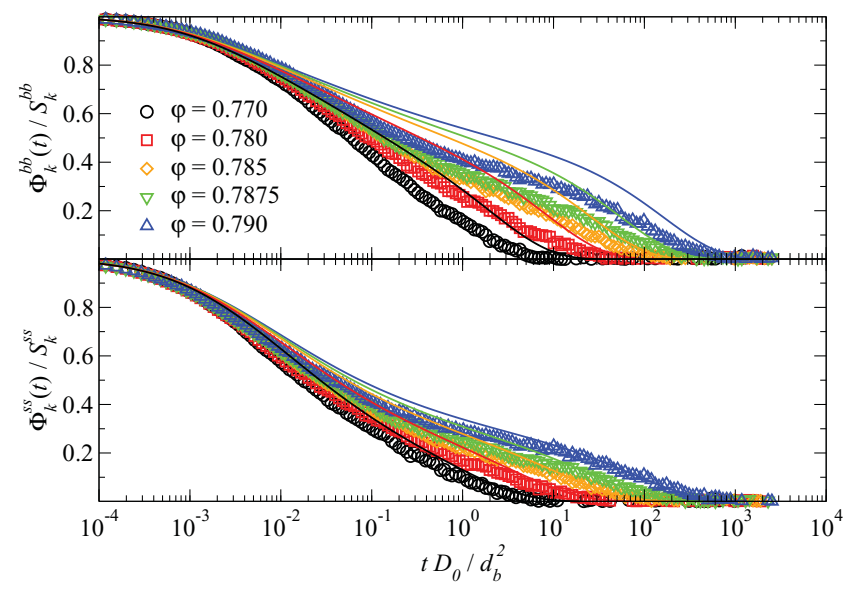

FIG. 16. (Color online) Normalized collective correlators from BD simulations for $\delta=5 / 7, x_{s}=0.5$, and $k d_{b}=13.0$. Solid lines show corresponding MCT results where the packing fractions have been mapped according to Eq. (38).

the BD-simulated correlation functions for $\varphi \geqslant 0.78$. Note, especially, that in this parameter regime, MCT describes the final part of the $\alpha$-relaxation process correctly also on the quantitative level.

Of course, the grade of quantitative compliance of our $\mathrm{BD}$ simulation and MCT results is also dependent on the wave number. We demonstrate this in Fig. 15 for $\Phi_{k}^{\alpha \alpha}(t) / S_{k}^{\alpha \alpha}$ at $k d_{b}=6.4$ and in Fig. 16 for $\Phi_{k}^{\alpha \alpha}(t) / S_{k}^{\alpha \alpha}$ at $k d_{b}=13.0$. For instance, MCT strongly overestimates the plateau values and also the $\alpha$-relaxation times for $\Phi_{k}^{b b}(t) / S_{k}^{b b}$ at both $k d_{b}=6.4$ and $k d_{b}=13.0$. For $\Phi_{k}^{s s}(t) / S_{k}^{s s}$ at $k d_{b}=6.4$, in contrast, MCT underestimates both the plateaus and the $\alpha$-relaxation times. For $\Phi_{k}^{s s}(t) / S_{k}^{s s}$ at $k d_{b}=13.0, \mathrm{MCT}$ slightly overestimates the plateaus but describes the $\alpha$ relaxation processes excellently. All these data indicate a connection between the (over-)underestimation of the plateaus and the (over-)underestimation of the $\alpha$-relaxation times by MCT.

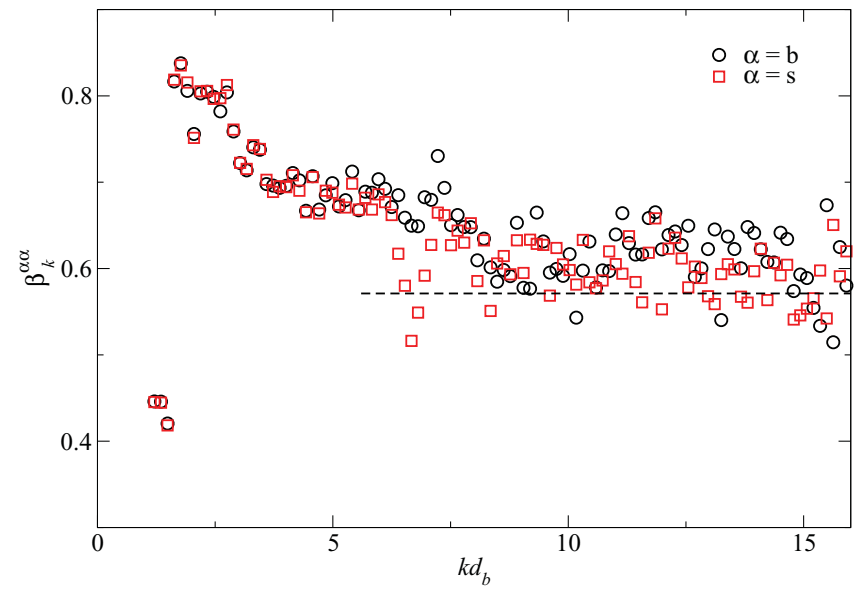

FIG. 17. (Color online) Kohlrausch stretching exponent $\beta_{k}^{\alpha \alpha}$ for big [(black) circles] and small [(red) squares] particles at $\delta=5 / 7$, $x_{s}=0.5$, and $\varphi=0.79$. The MCT von Schweidler exponent $b=$ 0.5571 is indicated by the horizontal dashed (black) line. 
For completeness, in Fig. 17 we show the Kohlrausch stretching exponent for small and big particles. For large $k d_{b}$ the exponent converges as $\beta_{k}^{\alpha \alpha} \rightarrow 0.6 \pm 0.05$. Unfortunately, noisy data prevent a more precise determination of the high $-k d_{b}$ limit. Nevertheless, the high- $k d_{b}$ limit is in good accordance with MCT's von Schweidler exponent, Eq. (33), calculated to $b=0.571$ with simulated structure factors.

\section{BEYOND THE MCT GLASS TRANSITION}

Figure 18 shows an example of three correlators with packing fractions above the MCT glass transition point. Clearly, one can see that the plateaus of the correlators increase with $\varphi$. This leads to intersecting correlators and proves that the correlators no longer collapse on a master function when they are rescaled by their relaxation times. Furthermore, the correlators above $\varphi \gtrsim \varphi^{c} \approx 0.7948$ show relaxation processes not captured in MCT that make the correlators beyond the MCT glass transition point decay to 0 for long times.

The correlator at $\varphi=0.81$ does not show a relaxation within the time window accessible in our BD simulations. In the time window accessible by Newtonian dynamics a relaxation time could be extrapolated by fitting a Kohlrausch function to the coherent correlator at $k d_{b}=8.4$. These correlators were obtained by equilibrating 150 independent systems with Newtonian dynamics up to $\approx 20 \%$ of the structural relaxation time measured with the Kohlrausch fit. After performing the Newtonian equilibration we do not see any dependence on the time origin for correlation functions in the time window accessible by BD. Especially, the plateau values do not change any more. However, the onset of a final decay (Fig. 18) can be assumed, as the curves show a slight curvature for large times.

At this point we want to stress that even though the decay to 0 is not included in MCT, MCT still gives an explanation for the rise of the NEPs above the glass transition (see also Ref. [35]).

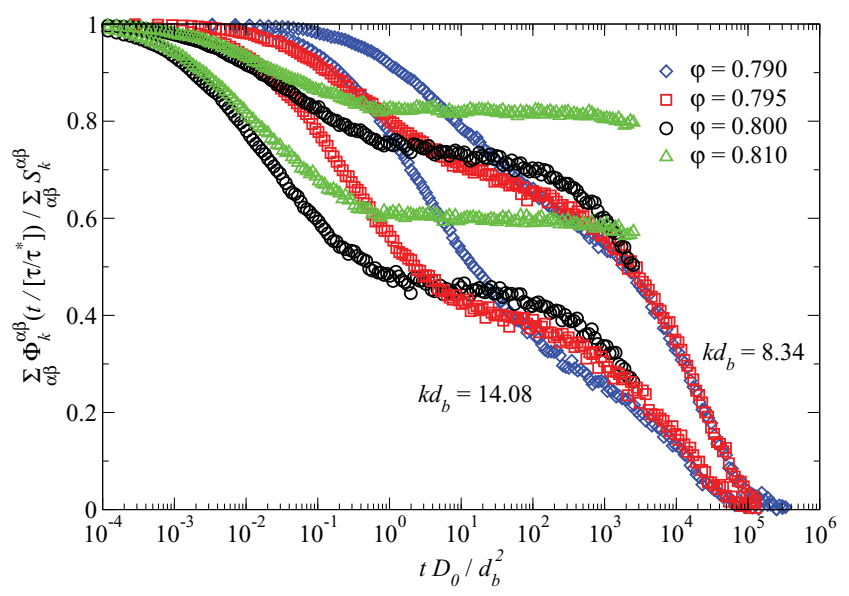

FIG. 18. (Color online) Three correlators above the MCT glass transition point at $\delta=5 / 7, x_{s}=0.5, \varphi=0.81, \varphi=0.80$, and $\varphi=0.795$, together with the highest liquid density at $\varphi=0.79$. The correlators violate the scaling property shown in Fig. 12. The plateau values increase upon going more deeply into the glass.

\section{SUMMARY AND CONCLUSIONS}

We have performed BD simulations for binary mixtures of hard disks in 2D to test systematically the predictions of MCT for this specific model system. Such a systematic test comparing collective density correlation functions from both approaches has not been carried out previously for a 2D model system.

Our main result is that MCT seems to be capable of capturing many qualitative features of the glass transition behavior of binary hard-disk mixtures in 2D. Particularly, all four mixing effects predicted by MCT for binary hard disks in $2 \mathrm{D}$ are indeed observable in our BD simulations. Furthermore, we have demonstrated the validity of the factorization theorem given by Eq. (22) and the $\alpha$-scaling law given by Eqs. (29) and (30). We have found that the MCT exponent $\gamma=2.4969$ describes excellently the dependence of the $\alpha$-relaxation process on the packing fraction $\varphi$ in the liquid regime close to the glass transition. Furthermore, MCT describes very well the qualitative $t$ and $\varphi$ dependences of the BD-simulated collective correlation functions close to the glass transition if one rescales the packing fraction according to Eq. (38). All these facts strongly support the quality of MCT in 2D. The range of validity of the asymptotic scaling law is very similar to previous results for hard spheres in 3D.

Going beyond the extrapolated glass transition at $\varphi_{\text {sim }}^{c} \cong$ 0.7948 , our BD simulation correlators lose the $\alpha$-scaling property, which results in intersecting correlators when they are rescaled by their relaxation times.

On the quantitative level, we have found some discrepancies between MCT and our BD simulation results. For instance, MCT underestimates the absolute value for the critical packing fraction for vitrification by about $15 \%$, similar to previous results for hard spheres in 3D. It seems to be possible to suppress the influence of this error on time-dependent quantities by introducing a linear map according to Eq. (38), at least for packing fractions close to the glass transition. We have found quantitative deviations of the $\mathrm{BD}$ simulation results for the NEPs and the critical amplitudes from the corresponding MCT results. For a better understanding of these deviations it would be necessary to investigate the leading-order corrections to the asymptotic scaling laws presented in Sec. VD. This, however, would go far beyond the scope of our present study.

In the present paper we have verified mixing scenarios predicted by MCT for binary mixtures of hard disks in 2D. Recently, mixing effects on the glass transition have also been investigated systematically in the framework of MCT for binary mixtures of dipolar point particles in 2D [21]. As a future project it would be worthwhile also to test the MCT results systematically by $\mathrm{BD}$ simulations.

\section{ACKNOWLEDGMENTS}

We thank Th. Voigtmann for discussions and hints concerning the treatment of binary mixtures. Special thanks go to M. Fuchs and R. Schilling, who gave us the opportunity to conduct this project on our own. 
[1] K. Binder and W. Kob, Glassy Materials and Disordered Solids (World Scientific, Singapore, 2005).

[2] U. Bengtzelius, W. Götze, and A. Sjölander, J. Phys. C 17, 5915 (1984).

[3] E. Leutheusser, Phys. Rev. A 29, 2765 (1984).

[4] W. Götze, Complex Dynamics of Glass-Forming Liquids (Oxford University Press, Oxford, 2009).

[5] W. Götze and L. Sjögren, Rep. Prog. Phys. 55, 241 (1992).

[6] W. Götze and Th. Voigtmann, Phys. Rev. E 67, 021502 (2003).

[7] S. I. Henderson, T. C. Mortensen, S. M. Underwood, and W. van Megen, Physica A 233, 102 (1996).

[8] S. R. Williams and W. van Megen, Phys. Rev. E 64, 041502 (2001).

[9] T. Voigtmann, Phys. Rev. E 68, 051401 (2003).

[10] G. Foffi, W. Götze, F. Sciortino, P. Tartaglia, and Th. Voigtmann, Phys. Rev. Lett. 91, 085701 (2003).

[11] G. Foffi, W. Götze, F. Sciortino, P. Tartaglia, and Th. Voigtmann, Phys. Rev. E 69, 011505 (2004).

[12] B. Schmid and R. Schilling, Phys. Rev. E 81, 041502 (2010).

[13] A. Ikeda and K. Miyazaki, Phys. Rev. Lett. 104, 255704 (2010).

[14] F. Ebert, P. Dillmann, G. Maret, and P. Keim, Rev. Sci. Instrum. 80, 083902 (2009).

[15] H. König, R. Hund, K. Zahn, and G. Maret, Eur. Phys. J. E 18, 287 (2005).

[16] S. Mazoyer, F. Ebert, G. Maret, and P. Keim, Europhys. Lett. 88, 66004 (2009).

[17] L. Santen and W. Krauth, Nature 405, 550 (2000).
[18] M. Bayer, J. M. Brader, F. Ebert, M. Fuchs, E. Lange, G. Maret, R. Schilling, M. Sperl, and J. P. Wittmer, Phys. Rev. E 76, 011508 (2007).

[19] T. Franosch, M. Fuchs, W. Götze, M. R. Mayr, and A. P. Singh, Phys. Rev. E 55, 7153 (1997).

[20] D. Hajnal, J. M. Brader, and R. Schilling, Phys. Rev. E 80, 021503 (2009).

[21] D. Hajnal, M. Oettel, and R. Schilling, J. Non-Cryst. Solids 357, $302(2011)$.

[22] A. Scala, T. Voigtmann, and C. De Michele, J. Chem. Phys. 126, 134109 (2007).

[23] O. Henrich, F. Weysser, M. E. Cates, and M. Fuchs, Philos. Trans. R. Soc. A 367, 5033 (2009).

[24] M. Fuchs, W. Götze, I. Hofacker, and A. Latz, J. Phys. Condens. Matter 3, 5047 (1991).

[25] T. Franosch and Th. Voigtmann, J. Stat. Phys. 109, 237 (2002).

[26] V. I. Arnol'd, Catastrophe Theory, 3rd ed. (Springer, Berlin, 1992).

[27] T. Voigtmann, Ph.D. thesis, TU München (2002).

[28] D. Hajnal and M. Fuchs, Eur. Phys. J. E 28, 125 (2009).

[29] T. Gleim, W. Kob, and K. Binder, Phys. Rev. Lett. 81, 4404 (1998).

[30] M. Fuchs, J. Non-Cryst. Solids 172-174, 241 (1994).

[31] O. Gräser, Diplomarbeit, Universität Konstanz (2006).

[32] D. Hajnal, Ph.D. thesis, Johannes Gutenberg-Universität Mainz (2010).

[33] T. Gleim and W. Kob, Eur. Phys. J. B 13, 83 (2000).

[34] E. Flenner and G. Szamel, Phys. Rev. E 72, 011205 (2005).

[35] J. Reinhardt, F. Weysser, and M. Fuchs, Phys. Rev. Lett. 105, 199604 (2010). 